\title{
Existence and Equivalence Theorems of Automorphic Systems
}

\author{
By \\ Kazushige UENo
}

\section{Introduction}

Systems of differential equations which admit pseudo-groups are seemed to be interesting to us.

Roughly speaking, a system of differential equations $(A)$ is said to admit a pseudo-group $\Gamma$ if, for each solution $s$ of $(A), \phi \circ s$ is also a solution of $(A)$ for any $\phi \in \Gamma$.

For the study of such systems, it is fundamental to investigate $\Gamma$ automorphic systems. For a pseudo-group $\Gamma$, a system of differential equations admitting $\Gamma$ is called a $\Gamma$-automorphic system if any solution of the system is of the form $\phi \circ s, \phi \in \Gamma$, where $s$ is a particular solution.

For these systems of differential equations, we shall pose the following two problems: (1) How generally are there $\Gamma$-automorphic systems? (2) What are necessary and sufficient conditions in order that $\Gamma$-automorphic systems $(A)_{1}$ and $(A)_{2}$ are isomorphic?

The purpose of this paper is to give some answers to the problems (1) and (2) for complete pseudo-groups.

As to the problem (1), it is the conclusion that, under certain regularity and completeness conditions for a pseudo-group $\Gamma$ on a manifold $Q$, there exists a $\Gamma$-automorphic system for any local submersion $s$ of a manifold $N$ to $Q$ such that $s$ is a particular solution of the system (Theorem 6.1).

As to the problem (2), we can give a necessary and sufficient condition for $\Gamma$-automorphic systems whose solutions are local submersions of $N$ to $Q$ (Theorem 7.1).

Communicated by N. Shimada, January 17, 1975. Revised May 6, 1975 
The problem (2) is a special case of equivalence problems of differential equations which appear naturally, when we consider integration problems according to Lie's integration program. Lie's program is not stated explicitly anywhere in his papers. But we can perceive it enough through his works. This paper is strongly affected by his thought.

In $\S 1$, we state some properties of differential invariants of pseudogroups.

In $\S 2$, we study about the existence of $\Gamma$-automorphic systems for complete pseudo-groups.

In $\S 3$, we state a necessary and sufficient condition for two automorphic systems to be isomorphic.

In $\S 4$, we give an interpretation of completeness of pseudo-groups.

In $\S 5$, we give such examples of $\Gamma$-automorphic systems that they are isomorphic. In Example 1, $\Gamma$ is of finite type and in Example 2, $\Gamma$ is of infinite type.

\section{§1. Differential Invariants}

1. Throughout this paper, we assume the differentiability of class $C^{\omega}$. Let $Q$ be a manifold and let $\mathscr{A}$ denote the sheaf of germs of all local vector fields on $Q$. We set $\mathscr{A}_{0}(z)=\left\{X \in \mathscr{L}(z) \mid X_{z}=0\right.$ where $X_{z}$ is the value of $X$ at $z$. $\}$ and, for $k \geqq 0$, we set $\mathscr{A}_{k+1}(z)=\left\{X \in \mathscr{A}_{k}(z) \mid\right.$ $\left.j_{z}^{k+1}(X)=0\right\}$ where $j_{z}^{k+1}(X)$ is the $(k+1)$-jet of $X$ at $z$. We set $A(z)$ $=\lim _{k \rightarrow \infty} \mathscr{A}(z) / \mathscr{A}_{k}(z)$. Then there exists a natural homomorphism $\mathbb{F}_{z}$ of $\mathscr{A}(z)$ to $A(z)$. Let $\mathscr{L}$ be a weak Lie algebra sheaf on $Q$ (as for its definition, refer to [4]) and we set $\mathscr{L}_{k}(z)=\mathscr{L}(z) \cap \mathscr{A}_{k}(z)$. Then $L(z)$ $=\lim _{k \rightarrow \infty} \mathscr{L}(z) / \mathscr{L}_{k}(z)$ is a Lie subalgebra of $A(z)$.

Definition 1.1. A weak Lie algebra sheaf $\mathscr{L}$ is said to be regular if $\operatorname{dim} \mathscr{L}_{k}(z) / \mathscr{L}_{k+1}(z)$ depends only on $k$, which we denote by $d_{k}(\mathscr{L})$ $(k=-1,0, \ldots)$ where $\mathscr{L}_{-1}(z)=\mathscr{L}(z)$.

Definition 1.2. A weak Lie algebra sheaf $\mathscr{L}$ is called a Lie algebra sheaf if it satisfies the following property;

(*) For a vector field $X$ on an open set $U \subset Q$ and any point $z \in U$, if $F_{z}(X(z)) \in L(z)$ where $X(z)$ is the germ of $X$ at $z$, then $X$ is 
a local cross-section of $\mathscr{L}$.

We denote by $J^{l}(N, Q)$ the space of $l$-jets of local maps of $N$ to $Q$ and, if $\operatorname{dim} N \geqq \operatorname{dim} Q$, we denote by $\tilde{J}^{l}(N, Q)$ the space of $l$-jets of local maps of $N$ to $Q$ which are of maximal rank. Then $\tilde{J}^{l}(N, Q)$ is open in $J^{l}(N, Q)$. For any $p \in J^{l}(N, Q)$, we denote by $\alpha(p)(\operatorname{resp} . \beta(p))$ the source of $p$ (resp. the target of $p$ ). Then we can regard $\alpha$ or $\beta$ as the projection of $J^{l}(N, Q)$ onto $N$ or $Q$ respectively.

Let $X$ be any local cross-section of a weak Lie algebra sheaf $\mathscr{L}$ and let $\phi_{t}$ be the local 1-parameter group of local transformations generated by $X$. We assume that $X$ is defined on $U$. For any $p \in \beta^{-1}(U)$ $\subset J^{l}(N, Q)$, we have a map $s: N \supset V_{\alpha(p)} \rightarrow Q$ such that $p=j_{\alpha(p)}^{l}(s)$. We set $\phi_{t}^{(l)}(p)=j_{\alpha(p)}^{l}\left(\phi_{t} \circ\right)$. Then $\phi_{t}^{(l)}$ is a local 1-parameter group of local transformations and induces a local vector field $X^{(l)}$ defined on $\beta^{-1}(U)$. Here we set $J^{0}(N, Q)=N \times Q$. We set $\mathscr{L}^{(l)}(p)=\left\{X^{(l)}(p) \mid X\right.$ is a local cross-section defined on a neighbourhood of $\beta(p)\}$. Then, if $\operatorname{dim} N \geqq$ $\operatorname{dim} Q$, we have

Lemma 1.1. For $l \geqq 0, \mathscr{L}^{(l)}=\underset{p \in \mathcal{J}^{l}(N, Q)}{\bigcup} \mathscr{L}^{(l)}(p)$ is a regular weak Lie algebra sheaf on $\tilde{J}^{l}(N, Q)$ if $\mathscr{L}^{p \in \tilde{J}^{l}(N, Q)}$ is a regular weak Lie algebra sheaf on $Q$.

Proof. It is easy to see that $\mathscr{L}^{(l)}$ is a sheaf with Lie algebra structure. We shall show that $\mathscr{L}^{(l)}$ is regular. Let $p=j_{x}^{l}(f) \in \tilde{J}^{l}(N, Q)$. Since $f$ is of maximal rank, it is clear that the map $\sigma^{(l)}: \mathscr{L}(\beta(p)) \rightarrow \mathscr{L}^{(l)}(p)$ with $\sigma^{(l)}(X(\beta(p)))=X^{(l)}(p)$ is a Lie algebra isomorphism. Moreover $\sigma^{(l)}$ satisfies $\sigma^{(l)}\left(\mathscr{L}_{l+h}(\beta(p))\right)=\mathscr{L}_{h}^{(l)}(p)(h=-1,0,1,2, \ldots)$ where $\mathscr{L}_{1}^{(l)}(p)=\mathscr{L}^{(l)}(p)$. This implies that $\operatorname{dim} \mathscr{L}^{(l)}(p) / \mathscr{L}_{0}^{(l)}(p)=\sum_{k=-1}^{l-1} d_{k}(\mathscr{L})$ and $\operatorname{dim} \mathscr{L}_{h}^{(l)}(p) / \mathscr{L}_{h+1}^{(l)}(p)$ $=d_{l+h}(\mathscr{L})(h=0,1, \ldots)$. This implies that $\mathscr{L}^{(l)}$ is regular on $\tilde{J}^{l}(N, Q)$ if $\mathscr{L}$ is regular.

\section{Let $\mathscr{L}$ be a weak Lie algebra sheaf on $Q$.}

Definition 2.1. Let $\varphi$ be a function defined locally at $j_{x}^{l}(f) \in J^{l}(N$, $Q) . \quad \varphi$ is called a differential invariant of $\mathscr{L}$ at $j_{x}^{l}(f)$, if, for any local cross-section $X$ of $\mathscr{L}$ at $f(x) \in Q$, we have $X^{(l)} \cdot \varphi=0$.

We assume that $\mathscr{L}$ is regular and $\operatorname{dim} N \geqq \operatorname{dim} Q$. We set $D_{p}^{(l)}$ 
$=\mathscr{L}^{(l)}(p) / \mathscr{L}_{0}^{(l)}(p), p \in \tilde{J}^{l}(N, Q)$. Then by Lemma 1.1 , the assignment $\tilde{J}^{l}(N, Q) \ni p \rightarrow D_{p}^{(l)}$ gives an involutive distribution on $\tilde{J}^{l}(N, Q)$ which we denote by $D_{\mathscr{Q}}^{(l)}(l \geqq 0)$. Here we also set $\tilde{J}^{0}(N, Q)=N \times Q$.

Definition 2.2. A fundamental system of first integrals of $D_{\mathscr{L}}^{(l)}$ at $p \in \tilde{J}^{l}(N, Q)$ is called a fundamental system of differential invariants of $\mathscr{L}$ at $p$.

Note that any first integral of $D_{\mathscr{Q}}^{(l)}$ at $p \in \tilde{J}^{l}(N, Q)$ is a differential invariant of $\mathscr{L}$ at $p$.

Let $f$ be a local map of $N \supset U$ onto $V \subset Q$ of maximal rank. We denote by $\left\{x_{1}, \ldots, x_{n}\right\}$ (resp. $\left\{z_{1}, \ldots, z_{q}\right\}$ ) a coordinate system on $U$ (resp. $V)$ and by $\left\{x_{i}(1 \leqq i \leqq n), z_{j}(1 \leqq j \leqq q), p_{j_{1} \ldots j_{k}}^{\lambda}\left(1 \leqq \lambda \leqq q, 1 \leqq j_{1}, \ldots, j_{k} \leqq n, 1 \leqq k\right.\right.$ $\leqq l)\}$ a coordinate system on a neighbourhood $U^{l}=\left(\rho_{0}^{l}\right)^{-1}(U \times V) \subset J^{l}(N$, $Q)$ of $j_{x}^{l}(f)$ where $\rho_{k}^{l}, l>k$, is the projection of $\tilde{J}^{l}(N, Q)$ onto $\tilde{J}^{k}(N, Q)$.

For a function $\phi$ on an open subset $W \subset U^{l}$, we set $\partial_{i}^{\sharp} \phi=\frac{\partial \phi}{\partial x_{i}}+$ $\sum_{\lambda} p_{i}^{\lambda} \frac{\partial \phi}{\partial z_{\lambda}}+\cdots+\sum_{j_{1}, \ldots, j_{l}} p_{j_{1} \ldots j_{l} i}^{\lambda} \frac{\partial \phi}{\partial p_{j_{1} \ldots j_{l}}^{\lambda}}$. Then $\partial_{i}^{\sharp} \phi$ is a function defined

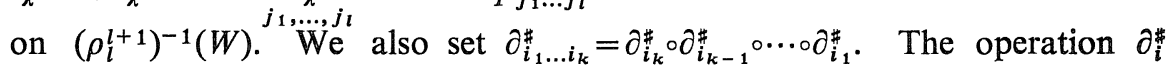
possesses the following properties.

Lemma 2.1. For any $1 \leqq i \leqq n$ and $l \geqq 1$, we have $\partial_{i}^{\sharp}\left(\rho_{l-1}^{l}\right)^{*}=\left(\rho_{l}^{l+1}\right)^{*} \partial_{i}^{\#}$.

Proof. Let $y$ be any function defined on a neighbourhood of $p \in$ $U^{l-1}$. Then we have

$$
\begin{aligned}
\partial_{i}^{\sharp}\left(\rho_{l-1}^{l}\right)^{*} y= & \partial_{i}^{\sharp}\left(y \circ \rho_{l-1}^{l}\right) \\
= & \frac{\partial\left(y \circ \rho_{l-1}^{l}\right)}{\partial x_{i}}+\sum_{\lambda} p_{i}^{\lambda} \frac{\partial\left(y \circ \rho_{l-1}^{l}\right)}{\partial z_{\lambda}}+\cdots \\
& \quad+\sum_{j_{1}, \ldots, j_{l}} p_{j_{1} \ldots j_{l} i}^{\lambda} \frac{\partial\left(y \circ \rho_{l-1}^{l}\right)}{\partial p_{j_{1} \ldots j_{l}}^{\lambda}}
\end{aligned}
$$

On the other hand we have

$$
\begin{aligned}
\left(\rho_{l}^{l+1}\right)^{*} \partial_{i}^{\sharp} y= & \left(\rho_{l}^{l+1}\right)^{*}\left\{\frac{\partial y}{\partial x_{i}}+\Sigma p_{i}^{\lambda} \frac{\partial y}{\partial z_{\lambda}}+\cdots\right. \\
& \left.+\sum_{\substack{\lambda \\
j_{1}, \ldots, j_{l-1}}} p_{j_{1} \ldots j_{l-1} i}^{\lambda} \frac{\partial y}{\partial p_{j_{1} \ldots j_{l-1}}^{\lambda}}\right\} .
\end{aligned}
$$


Now we have clearly

$$
\begin{gathered}
\frac{\partial\left(y \circ \rho_{l-1}^{l}\right)}{\partial x_{i}}=\left(\rho_{l}^{l+1}\right)^{*} \frac{\partial y}{\partial x_{i}}, p_{i}^{\lambda} \frac{\partial\left(y \circ \rho_{l-1}^{l}\right)}{\partial z_{\lambda}}=\left(\rho_{l}^{l+1}\right)^{*} p_{i}^{\lambda} \frac{\partial y}{\partial z_{\lambda}}, \\
\ldots, p_{j_{1} \ldots j_{l-1} i}^{\lambda} \frac{\partial\left(y \circ \rho_{l-1}^{l}\right)}{\partial p_{j_{1} \ldots j_{l-1}}^{\lambda}}=\left(\rho_{l}^{l+1}\right)^{*} p_{j_{1} \ldots j_{l-1} i}^{\lambda} \frac{\partial y}{\partial p_{j_{1} \ldots j_{l-1}}^{\lambda}}
\end{gathered}
$$

and $\frac{\partial\left(y \circ \rho_{l-1}^{l}\right)}{\partial p_{j_{1} \ldots j_{l}}^{\lambda}}=0$

These imply that we get

$$
\partial_{i}^{\sharp}\left(\rho_{l-1}^{l}\right)^{*}=\left(\rho_{l}^{l+1}\right)^{*} \partial_{i}^{\#} .
$$

Lemma 2.2. Let $y$ be any function defined on a neighbourhood of $p \in U^{l}$ and let $\phi$ be any local diffeomorphism of $Q \supset U^{\prime}$ to a neighbourhood $U_{\beta(p)}$ of $\beta(p)$. Then we have $\left(\phi^{(l+1)}\right)^{*} \partial_{i}^{\sharp} y=\partial_{i}^{\sharp}\left(\left(\phi^{(l)}\right)^{*} y\right)$.

Proof. We have

$$
\begin{gathered}
\frac{\partial\left(y \circ \phi^{(l)}\right)}{\partial z_{\lambda}}=\sum_{\mu} \frac{\partial y}{\partial z_{\mu}}\left(\phi^{(l)}\right) \frac{\partial z_{\mu}\left(\phi^{(l)}\right)}{\partial z_{\lambda}}+\sum_{\mu, j} \frac{\partial y}{\partial p_{j}^{\mu}}\left(\phi^{(l)}\right) \frac{\partial p_{j}^{\mu}\left(\phi^{(l)}\right)}{\partial z_{\lambda}} \\
+\cdots+\sum_{\substack{\mu \\
j_{1}, \ldots, j_{l}}} \frac{\partial y}{\partial p_{j_{1} \ldots j_{l}}^{\mu}}\left(\phi^{(l)}\right) \frac{\partial p_{j_{1} \ldots j_{l}}^{\mu}\left(\phi^{(l)}\right)}{\partial z_{\lambda}}
\end{gathered}
$$

and

$$
\begin{aligned}
\frac{\partial\left(y \circ \phi^{(l)}\right)}{\partial p_{j_{1} \ldots j_{k}}^{\lambda}}= & \sum_{\mu} \frac{\partial y}{\partial z_{\mu}}-\left(\phi^{(l)}\right) \frac{\partial z_{\mu}\left(\phi^{(l)}\right)}{\partial p_{j_{1} \ldots j_{k}}^{\lambda}}+\sum_{\mu, j} \frac{\partial y}{\partial p_{j}^{\mu}}\left(\phi^{(l)}\right) \frac{\partial p_{j}^{\mu}\left(\phi^{(l)}\right)}{\partial p_{j_{1} \ldots j_{k}}^{\lambda}} \\
& +\cdots+\sum \frac{\partial y}{\partial p_{j_{1} \ldots j_{l}}^{\mu}}\left(\phi^{(l)}\right) \frac{\partial p_{j_{1} \ldots j_{l}}^{\mu}\left(\phi^{(l)}\right)}{\partial p_{j_{1} \ldots j_{k}}^{\lambda}} .
\end{aligned}
$$

Therefore we get

$$
\begin{aligned}
\partial_{i}^{\#}\left(y \circ \phi^{(l)}\right)= & \frac{\partial\left(y \circ \phi^{(l)}\right)}{\partial x_{i}}+\sum_{\lambda} p_{i}^{\lambda} \frac{\partial\left(y \circ \phi^{(l)}\right)}{\partial z_{\lambda}} \\
& +\cdots+\sum_{\substack{\lambda \\
j_{1}, \ldots, j_{1},}} p_{j_{1} \ldots j_{l} i}^{\lambda} \frac{\partial\left(y \circ \phi^{(l)}\right)}{\partial p_{j_{1} \ldots j_{l}}^{\lambda}}
\end{aligned}
$$




$$
\begin{aligned}
= & \frac{\partial\left(y \circ \phi^{(l)}\right)}{\partial x_{i}}+\sum_{\mu}\left(\sum_{\lambda} p_{i}^{\lambda} \frac{\partial z_{\mu}\left(\phi^{(l)}\right)}{\partial z_{\lambda}}\right. \\
& \left.+\cdots+\sum_{\substack{\lambda \\
j_{i}, \ldots, j_{l}}} p_{j_{1} \ldots j_{l} i}^{\lambda} \frac{\partial z_{\mu}\left(\phi^{(l)}\right)}{\partial p_{j_{1} \ldots j_{l}}^{\lambda}}\right) \frac{\partial y}{\partial z_{\mu}}\left(\phi^{(l)}\right) \\
& +\cdots+\sum_{j_{1}, \ldots, j_{l}}\left(\sum_{\lambda} p_{i}^{\lambda} \frac{\partial p_{j_{1} \ldots j_{l}}^{\mu}\left(\phi^{(l)}\right)}{\partial z_{\lambda}}\right. \\
& \left.+\cdots+\sum_{\substack{\lambda \\
j_{1}, \ldots, j_{l}}} p_{j_{1} \ldots j_{l} i}^{\lambda} \frac{\partial p_{j_{1} \ldots j_{l}}^{\mu}\left(\phi^{(l)}\right)}{\partial p_{j_{1} \ldots j_{l}}^{\lambda}}\right) \\
& \times \frac{\partial y}{\partial p_{j_{1} \ldots j_{l}}^{\mu}}\left(\phi^{(l)}\right),
\end{aligned}
$$

and

$$
\begin{gathered}
\frac{\partial\left(y \circ \phi^{(l+1)}\right)}{\partial x_{i}}=\frac{\partial\left(y \circ \phi^{(l)}\right)}{\partial x_{i}}, \frac{\partial y}{\partial z_{\mu}}\left(\phi^{(l+1)}\right)=\frac{\partial y}{\partial z_{\mu}}\left(\phi^{(l)}\right) \\
\ldots, \frac{\partial y}{\partial p_{j_{1} \ldots j_{l}}^{\mu}}\left(\phi^{(l+1)}\right)=\frac{\partial y}{\partial p_{j_{1} \ldots j_{l}}^{\mu}}\left(\phi^{(l)}\right) \\
p_{i}^{\mu}\left(\phi^{(l+1)}\right)=\sum_{\lambda} p_{i}^{\lambda} \cdot \frac{\partial z_{\mu}\left(\phi^{(l)}\right)}{\partial z_{\lambda}} \\
+\cdots+\sum_{\substack{\lambda \\
j_{1}, \ldots, j_{l}}} p_{j_{1} \ldots j_{l} i}^{\lambda_{l}} \frac{\partial z_{\mu}\left(\phi^{(l)}\right)}{\partial p_{j_{1} \ldots j_{l}}^{\lambda}}, \cdots \\
p_{j_{1} \ldots j_{l} i}^{\mu}\left(\phi^{(l+1)}\right)=\sum_{\lambda} p_{i}^{\lambda} \cdot \frac{\partial p_{j_{1} \ldots j_{l}}^{\mu}\left(\phi^{(l)}\right)}{\partial z_{\lambda}} \\
+\cdots+\sum p_{j_{1} \ldots j_{l} i}^{\lambda} \cdot \frac{\partial p_{j_{1} \ldots j_{l}}^{\mu}\left(\phi^{(l)}\right)}{\partial p_{j_{1} \ldots j_{l}}^{\lambda}}
\end{gathered}
$$

This implies $\partial_{i}^{\sharp}\left(\left(\phi^{(l)}\right)^{*} y\right)=\left(\phi^{(l+1)}\right) * \partial_{i}^{\sharp} y$.

The following Lemma is the infinitesimal form of Lemma 2.2.

Lemma 2.3. $X^{(l+1)} \cdot \partial_{i}^{\sharp} y=\partial_{i}^{\sharp}\left(X^{(l)} \cdot y\right)$ for any local function $y$ at $p \in \tilde{J}^{l}(N, Q)$ and any local vector field $X$ at $\beta(p)$.

The immediate consequence of Lemma 2.3 is 
Lemma 2.4. Let $\mathscr{L}$ be a regular weak Lie algebra sheaf on $Q$ and let $y$ be any differential invariant of $\mathscr{L}$ at $p^{l}=j_{x}^{l}(f) \in \tilde{J}^{l}(N, Q)$. Then $\partial_{i}^{\#} y$ is also a differential invariant of $\mathscr{L}$ at $p^{l+1}=j_{x}^{l+1}(f)$.

\section{§2. Automorphic Systems of Submersion Type}

3. Let $\Gamma$ be a pseudo-group on $Q$ (as for the definition of "pseudogroups", refer to [4]). Except for the following two definitions, we assume that $\operatorname{dim} N \geqq \operatorname{dim} Q$.

Definition 3.1. Let $(A)$ be a differential equation given on a neighbourhood $V^{k}$ of $p \in J^{k}(N, Q)$. (A) is said to be $\Gamma$-automorphic if the following conditions are satisfied:

(1) There exists a solution.

(2) For any solution $s: U \rightarrow Q$ and any element $\phi: W_{1} \rightarrow W_{2} \subset \beta\left(V^{k}\right)$ in $\Gamma$, if $s(U) \subset W_{1}, \phi \circ s$ is also a solution.

(3) For any two solutions $s_{i}: U_{i} \rightarrow Q(i=1,2), U_{i} \ni \alpha(p)$, there exists an element $\phi \in \Gamma$ such that $s_{2}=\phi \circ s_{1}$ on a neighbourhood $U \subset U_{1} \cap U_{2}$ of $\alpha(p)$.

Definition 3.2. Let $\left(B^{i}\right)$ be a differential equation on $V^{k^{i}} \subset J^{h^{i}}$ $(N, Q)(i=1,2) . \quad\left(B^{1}\right)$ is said to be isomorphic to $\left(B^{2}\right)$ if there exists a diffeomorphism $\psi$ of $\beta\left(V^{k^{1}}\right)$ onto $\beta\left(V^{k^{2}}\right)$ such that $s$ is a solution of $\left(B^{1}\right)$ if and only if $\psi \circ S$ is a solution of $\left(B^{2}\right)$.

4. First of all, in this article, we shall state Kuranishi's prolongation theorem.

Theorem (Kuranishi). Let $\left(M, M^{\prime}, \omega\right)$ be a fibred manifold and let $\phi^{l}$ be a differential equation of order $l$ on $\left(M, M^{\prime}, \omega\right), l \geqq l_{0}$. We assume the following:

(i) There exists a local cross-section $s_{0}$ of $\left(M, M^{\prime}, \omega\right)$ at $x_{0} \in M^{\prime}$ such that $s_{0}$ is a solution of $\phi^{l}$ for any $l \geqq l_{0}$.

(ii) $\Phi^{l+1} \supset p\left(\Phi^{l}\right)$ on a neighbourhood of $X^{l}=j_{x_{0}}^{l}(f)$ where $X^{l}$ is an ordinary integral point of $\Phi^{l}$ for any $l \geqq l_{0}$ and $p\left(\Phi^{l}\right)$ is the standard prolongation of $\Phi^{l}$. 
(iii) For a suitable open neighbourhood $U$ of $X^{I_{0}},\left(I \Phi^{l_{0}} \cap U, \alpha(U), \alpha\right)$ is a fibred manifold, where $I \Phi^{k}$ is the set of integral points of $\Phi^{k}$.

(iv) $\left(I \Phi^{l+1} \cap \mathscr{V}, I \Phi^{l} \cap \mathscr{V}^{\prime}, \rho^{l+1}\right)$ is a fibred manifold for a suitable open neighbourhood $\mathscr{V}$ (resp. $\mathscr{V}^{\prime}$ ) of $X^{l+1}$ (resp. $X^{l}$ ) for any $l \geqq l_{0}$. Then there exists an integer $l_{1}$ such that $\Phi^{l+1}$ and $p\left(\Phi^{l}\right)$ are equal in a neighbourhood of $X^{l+1}$ and such that $\Phi^{l}$ is involutive at $X^{l}$ for any $l \geqq l_{1}$.

As for the proof of this theorem, refer to [1].

Lemma 4.1. Let $\mathscr{L}$ be a regular weak Lie algebra sheaf on $Q$ and let $\left\{(B)_{k}\right\}_{k \geqq 1}$ be a sequence of differential equations satisfying the following conditions:

(1) $(B)_{k}$ is generated by functions $y_{j}^{k}-\lambda_{j}^{k}\left(1 \leqq j \leqq m_{k}\right)$ at $p^{k}=j_{x_{0}}^{k}(s)$ $\in \tilde{J}^{k}(N, Q)$ where $\left\{y_{j}^{k}\right\}_{j=1}^{m_{k}}$ is a fundamental system of differential invariants of $\mathscr{L}$ at $p^{k}$ and $\left\{\lambda_{j}^{k}\right\}_{j=1}^{m_{h}}$ is a family of functions locally defined at $x_{0} \in N$.

(2) $s$ is a solution of $(B)_{k}$ for any $k \geqq 1$.

Then there exists an integer $l_{0}$ such that $f$ is a solution of $(B)_{l_{0}}$ if and only if it is a solution of $(B)_{l}$ for any $l \geqq l_{0}$.

Proof. We shall show that there exists an integer $l_{1}$ such that $(B)_{l+1}$ and $p(B)_{l}$ are equal in a neighbourhood of $p^{l}$ and $(B)_{l}$ is involutive at $p^{l}$ for any $l \geqq l_{1}$. For any $l,\left\{x_{j}\right\}_{j=1}^{n}$ are considered as differential invariants of $\mathscr{L}$ at $p^{l}$. We may assume that $\left\{x_{1}, \ldots, x_{n}, y_{1}^{l}, \ldots, y_{m_{l}-n}^{l}\right\}$ is a fundamental system of differential invariants of $\mathscr{L}$ at $p^{l}$. Then $y_{1}^{l}$ $-\lambda_{1}^{l}, \ldots, y_{m_{l}-n}^{l}-\lambda_{m_{l}-n}^{l}$ are linearly independent and $I(B)_{l}$ is the set of points satisfying $y_{j}^{l}-\lambda_{j}^{l}=0\left(1 \leqq j \leqq m_{l}-n\right)$. This implies that $p^{l}$ is an ordinary integral point of $(B)_{l}, l \geqq 1$. For any differential invariant $y^{l-1}$ of $\mathscr{L}$ at $p^{l-1}, \partial_{i}^{\#} y^{l-1}$ is a differential invariant of $\mathscr{L}$ at $p^{l}$ by Lemma 2.4. Therefore we get $\partial_{i}^{\sharp} y^{l-1}=\varphi_{i}^{l-1}\left(y_{1}^{l}, \ldots, y_{m_{l}}^{l}\right)$ for a suitable function $\varphi_{i}^{l-1}$. Let $y^{l-1}-\lambda^{l-1}$ be a function in $(B)_{l-1}$ where $\lambda^{l-1}$ (resp. $y^{l-1}$ ) is a function locally defined at $x_{0}$ (resp. a differential invariant of at $\left.p^{l}\right)$. We have $\partial_{i}^{\#}\left(y^{l-1}-\lambda^{l-1}\right)=\partial_{i}^{\#} y^{l-1}-\partial_{i}^{\#} \lambda^{l-1}=\varphi_{i}^{l-1}\left(y_{1}^{l}, \ldots, y_{m_{l}}^{l}\right)-\partial_{i}^{\#} \lambda^{l-1}$ and $\partial_{i}^{\#} \lambda^{l-1}(x)=\varphi_{i}^{l-1}\left(y_{1}^{l}, \ldots, y_{m_{l}}^{l}\right)\left(j_{x}^{l}(s)\right)$. On the other hand, we have clearly $\varphi_{i}^{l-1}\left(y_{1}^{l}, \ldots, y_{m_{l}}^{l}\right)\left(j_{x}^{l}(s)\right)=\varphi_{i}^{l-1}\left(\lambda_{1}^{l}(x), \ldots, \lambda_{m_{l}}^{l}(x)\right)$. Therefore we get 
$\partial_{i}^{\#} \lambda^{l-1}=\varphi_{i}^{l-1}\left(\lambda_{1}^{l}, \ldots, \lambda_{m_{l}}^{l}\right)$, that is, $\partial_{i}^{\sharp}\left(y^{l-1}-\lambda^{l-1}\right)=\varphi_{i}^{l-1}\left(y_{1}^{l}, \ldots, y_{m_{l}}^{l}\right)-\varphi_{i}^{l-1}$ $\left(\lambda_{1}^{l}, \ldots, \lambda_{m_{l}}^{l}\right)$. Therefore we get easily $\partial_{i}^{\sharp}\left(y^{l-1}-\lambda^{l-1}\right)=\sum_{j=1}^{m_{l}-n} g_{j}^{l} \cdot\left(y_{j}^{l}-\lambda_{j}^{l}\right)$. Since $(B)_{l}$ is an ideal as a sheaf, this implies that $(B)_{l}$ contains $p(B)_{l-1}$. Since $s: U \rightarrow Q$ is a solution of $(B)_{k}$ for any $k \geqq 1$, if we set $\mathscr{U}=\alpha^{-1}(U)$ $\subset \tilde{J}^{k}(N, Q),\left(I(B)_{k} \cap \mathscr{U}, \alpha(\mathscr{U}), \alpha\right)$ is a fibred manifold. We set $s(k)=\left\{j_{x}^{k}(s) \mid\right.$ $x \in U\}$. Then $s(k)$ is a submanifold of $\tilde{J}^{k}(N, Q)$ and, since $x_{1}, \ldots, x_{n}$ are differential invariants of $\mathscr{L}, T_{p}(s(k)) \cap D_{p}^{(k)}=0, p \in s(k)$, where $D^{(k)}$ is the involutive distribution on $\tilde{J}^{k}(N, Q)$ induced from $\mathscr{L}$. Let $\mathscr{I}_{p}^{k}$ the maximal integral manifold of $D^{(k)}$ through $p \in s(k)$. We see that there exists a neighbourhood $\mathscr{V}^{k}$ of $p^{k}$ such that $\mathscr{I}_{p}^{k} \cap \mathscr{V}^{k}={ }^{\prime} \mathscr{J}_{p}^{k} \cap \mathscr{V}^{k}$ if $\left(\mathscr{I}_{p}^{k} \cap \mathscr{V}^{k}\right)$ $\cap\left({ }^{\prime} \mathscr{I}_{p}^{k} \cap \mathscr{V}^{k}\right) \neq \varnothing$. Since $T_{p}(s(k)) \cap D_{p}^{(k)}=0, \underset{p \in s(k)}{\bigcup} \mathscr{I}_{p}^{k} \cap \mathscr{V}^{k}$ is a manifold with $\operatorname{dim} \bigcup_{p \in s(k)} \mathscr{I}_{p}^{k} \cap \mathscr{V}^{k}=n+\operatorname{dim} D^{(k)}$. On the other hand, $\left\{x_{1}, \ldots, x_{n}, y_{1}^{k}, \ldots\right.$, $\left.y_{m_{k}-n}^{k}\right\}$ is a fundamental system of first integrals of $D^{(k)}$ at $p^{k}$ and $I(B)_{k}$ is the set of points satisfying $y_{1}^{k}-\lambda_{1}^{k}=0, \ldots, y_{m_{k}-n}^{k}-\lambda_{m_{k}-n}^{k}=0$. Therefore we have $\underset{p \in s(k)}{\bigcup} \mathscr{J}_{p}^{k} \cap \mathscr{V}^{k} \subset I(B)_{k}$ and $\operatorname{dim} I(B)_{k}=n+\operatorname{dim} D^{(k)}$. This implies that we have $\underset{p \in s(k)}{\cup} \mathscr{I}_{p}^{k} \cap \mathscr{V}^{k}=I(B)_{k} \cap \mathscr{V}^{k}$. It is easy to see that, for a suitable neighbourhood $\mathscr{V}$ (resp. $\mathscr{V}^{\prime}$ ) of $p^{l+1}$ (resp. $\left.p^{l}\right),\left(I(B)_{l+1} \cap \mathscr{V}\right.$, $\left.I(B)_{l} \cap \mathscr{V}^{\prime}, \rho_{l}^{l+1}\right)$ is a fibred manifold. Therefore, by Kuranishi's theorem, there exists an integer $l_{0}$ such that $(B)_{l+1}$ and $p(B)_{l}$ are equal in a neighbourhood of $p^{l}$ and $(B)_{l}$ is involutive at $p^{l}$ for any $l \geqq l_{0}$. This implies that $f$ is a solution of $(B)_{l_{0}}$ if and only if it is a solution of (B). for any $l \geqq l_{0}$. This completes the proof of Lemma 4.I.

Note that the integer $l_{0}$ is independent of the choice of a fundamental system of differential invariants $\left\{y_{j}^{l}\right\}_{j=1}^{m_{l}}$ of $\mathscr{L}$ at $p^{l}$.

Definition 4.1. Let $\bar{l}_{0}$ be the minimum integer such that $f$ is a solution of $(B)_{\bar{l}_{0}}$ if and only if $f$ is a solution of $(B)_{l}$ for any $l \geqq \bar{l}_{0} \cdot \bar{l}_{0}$ is called the order of $\mathscr{L}$ at $\left(x_{0}, s\right)$.

5. Let $\Gamma$ be a pseudo-group on $Q$ and let $\mathscr{L}_{\Gamma}$ be the maximal subsheaf of $\mathscr{A}$ such that each stalk of $\mathscr{L}_{\Gamma}$ is a Lie subalgebra of the corresponding stalk of $\mathscr{A}$ and such that the pseudo-group $\mathscr{P}\left(\mathscr{L}_{\Gamma}\right)$ generated by $\mathscr{L}_{\Gamma}$ is contained in $\Gamma$.

Definition 5.1. Let $\Gamma$ be a pseudo-group on $Q$ such that $\mathscr{L}_{\Gamma}$ is a 
regular Lie algebra sheaf. A neighbourhood $V^{k}$ of $j_{x}^{k}(f) \in \tilde{J}^{k}(N, Q)$ is called a $\Gamma$-neighbourhood if there is a fundamental system of differential invariants $\left\{y_{j}\right\}_{j=1}^{m_{k}}$ of $\mathscr{L}_{\Gamma}$ such that each $y_{j}$ is defined on $V^{k}$.

Let $f$ be a local diffeomorphism of $Q$ such that $f(x)=z$.

Definition 5.2. A pseudo-group $\Gamma$ on $Q$ is said to be complete at $(z, f)$ if the following conditions are satisfied:

(1) $\mathscr{L}_{\Gamma}$ is a regular Lie algebra sheaf.

(2) Let $\phi$ be a local diffeomorphism of $Q$ such that, for any integer $k \geqq 0, \phi^{(k)}$ maps an open subset $W^{k} \ni j_{x^{\prime}}^{k}\left(f^{\prime}\right)$ of a $\Gamma$-neighbourhood $V^{k}$ of $j_{x}^{k}(f)$ into $V^{k}$. Then a restriction ' $\phi$ of $\phi$ to a neighbourhood of $f^{\prime}\left(x^{\prime}\right)$ is in $\Gamma$ if and only if $\left(\phi^{(k)}\right)^{*} y_{j}^{k}=y_{j}^{k}$ on a neighbourhood ' $W^{k}$ of $j_{x^{\prime}}^{k}\left(f^{\prime}\right)$ for any integer $k \geqq 0$ and a fundamental system of differential invariants $\left\{y_{j}^{k}\right\}_{j=1}^{m_{k}}$ of $\mathscr{L}_{\Gamma}$ on $V^{k}$.

Lemma 5.1. Let $j_{x}^{l}(\rho) \in \tilde{J}^{l}(N, Q)$. Let $\phi$ be a local diffeomorphism of $Q$ such that $\phi^{(l)}$ maps an open subset $\tilde{W}^{l} \ni j_{x^{\prime}}^{l}\left(\rho^{\prime}\right)$ of a $\Gamma$-neighbourhood $\tilde{V}^{l}$ of $j_{x}^{l}(\rho)$ into $\tilde{V}^{l}$ for any integer $l \geqq 0$. We assume that $\Gamma$ is a pseudo-group on $Q$ which is complete at $(\rho(x), 1)$ where 1 is the identity of $Q$. Then ' $\phi \in \Gamma$ if and only if $\left(\phi^{(l)}\right)^{*} \eta_{j}^{l}=\eta_{j}^{l}$ on ' $\tilde{W}^{l}$ for any integer $l \geqq 0$ and a fundamental system of differential invariants $\left\{\eta_{j}^{l}\right\}_{j=1}^{n_{l}}$ of $\mathscr{L}_{\Gamma}$ on $\tilde{V}^{l}$. (The notations ' $\phi$ and ' $\tilde{W}^{l}$ are used like those of Definition 5.2.)

Proof. Since $\rho$ is a local map of maximal rank, there exist a neighbourhood $\mathscr{W}$ of $x \in N$ and a neighbourhood $\mathscr{U}$ of $\rho(x) \in Q$ such that $(N \cap \mathscr{W}, Q \cap \mathscr{U}, \rho)$ is a fibred manifold. Let $\iota$ be a local crosssection of $(N \cap \mathscr{W}, Q \cap \mathscr{U}, \rho)$. Then c induces a local map $\rho^{l}$ of $\tilde{J}^{l}(N$, $Q)$ to $\tilde{J}^{l}(Q, Q)$ such that, for a sufficiently small neighbourhood $\mathscr{W}^{l}$ of $j_{x}^{l}(\rho)$ and a sufficiently small neighbourhood $\mathscr{U}^{l}$ of $j_{\rho(x)}^{l}(\rho \circ \ell)=j_{\rho(x)}^{l}(1)$, $\left(\tilde{J}^{l}(N, Q) \cap \mathscr{W}^{l}, \tilde{J}^{l}(Q, Q) \cap \mathscr{U}^{l}, \rho^{l}\right)$ is a fibred manifold. We denote by $\psi_{1}^{(l)}\left(\right.$ resp. $\left.\psi_{2}^{(l)}\right)$ the prolongation of $\psi \in \Gamma$ to $\tilde{J}^{l}(N, Q)$ (resp. $\tilde{J}^{l}(Q, Q)$ ). We shall show that we have $\rho^{l} \circ \psi_{1}^{(l)}=\psi_{2}^{(l)} \circ \rho^{l}$. Let $j_{x}^{l}(g) \in \tilde{J}^{l}(N, Q) \cap \mathscr{W}^{l}$. Then we have $\rho^{l} \circ \psi_{1}^{(l)}\left(j_{x}^{l}(g)\right)=\rho^{l}\left(j_{x}^{l}(\psi \circ g)\right)=j_{\rho(x)}^{l}(\psi \circ g \circ \iota)$. On the other hand, we have $\psi_{2}^{(l)} \circ \rho^{l}\left(j_{x}^{l}(g)\right)=\psi_{2}^{(l)}\left(j_{\rho(x)}^{l}(g \circ \iota)\right)=j_{\rho(x)}^{l}(\psi \circ g \circ \iota)$. Therefore we get $\rho^{l} \circ \psi_{1}^{(l)}=\psi_{2}^{(l)} \circ \rho^{l}$. Now let $\left\{y_{1}^{l}, \ldots, y_{m_{l}}^{l}\right\}$ be a fundamental system of 
differential invariants of $\mathscr{L}_{\Gamma}$ at $j_{\rho(x)}^{l}(1) \in \tilde{J}^{l}(Q, Q)$. Then, by the commutativity $\rho^{l} \circ \psi_{1}^{(l)}=\psi_{2}^{(l)} \circ \rho^{l}$, we can see that $\left(\rho^{l}\right)^{*} y_{j}^{l}\left(1 \leqq j \leqq m_{l}\right)$ are differential invariants of $\mathscr{L}_{\Gamma}$ at $j_{x}^{l}(\rho) \in \tilde{J}^{l}(N, Q)$. It is clear that there exists a fundamental system of differential invariants $\left\{\eta_{1}^{l}, \ldots, \eta_{m_{l}}^{l}, \eta_{m_{l}+1}^{l}, \ldots, \eta_{n_{l}}^{l}\right\}$ of $\mathscr{L}_{\Gamma}$ at $j_{x}^{l}(\rho)$ such that $\eta_{j}^{l}=\left(\rho^{l}\right)^{*} y_{j}^{l}\left(1 \leqq j \leqq m_{l}\right)$. If $\phi$ is a local diffeomorphism stated above, then $\phi_{2}^{(l)}$ maps an open subset $W^{l} \ni j_{z}^{l}\left(f^{\prime}\right)\left(f^{\prime}(z)\right.$ $=\rho^{\prime}\left(x^{\prime}\right), f^{\prime}=\rho^{\prime} \circ$ ८) of a $\Gamma$-neighbourhood $V^{l}$ of $j_{\rho(x)}^{l}(1)$ into $V^{l}$ and clearly $\left(\phi_{2}^{(l)}\right)^{*} y_{j}^{l}=y_{j}^{l}\left(1 \leqq j \leqq m_{l}\right), l \geqq 0$, on ' $W^{l}$. Since $\Gamma$ is complete at $(\rho(x), 1)$, we get ' $\phi \in \Gamma$. If ' $\phi \in \Gamma$, then $\left(\phi_{2}^{(l)}\right)^{*} y_{j}^{l}=y_{j}^{l}\left(1 \leqq j \leqq m_{l}\right)$ on an open subset ' $W^{l} \ni j_{z}^{l}\left(f^{\prime}\right)$ of $V^{l}$ for any $l \geqq 0$. Therefore we get $\left(\phi_{1}^{(l)}\right)^{*} \eta_{j}^{l}=\eta_{j}^{l}\left(1 \leqq j \leqq m_{l}\right)$ on an open subset ' $\tilde{W}^{l} \ni j_{x^{\prime}}^{l}\left(\rho^{\prime}\right)$ of $\tilde{V}^{l}$. Let $\Gamma_{Q}$ be the pseudo-group of all local diffeomorphism of $Q$. Then it is easy to see that $\eta_{k}^{l}\left(m_{l}+1\right.$ $\left.\leqq k \leqq n_{l}\right)$ are chosen such that they are differential invariants of $\Gamma_{Q}$. Therefore, in particular, we get $\left(\phi_{1}{ }^{(l)}\right)^{*} \eta_{k}^{l}=\eta_{k}^{l}\left(m_{l}+1 \leqq k \leqq n_{l}\right)$ on an open subset ' $\tilde{W}^{l}$ of $\tilde{V}^{l}$.

Lemma 5.2. Let $\Gamma$ be a pseudo-group on $Q$ such that $\mathscr{L}_{\Gamma}$ is a regular Lie algebra sheaf. Let $j_{z_{0}}^{l}(1) \in \tilde{J}^{l}(Q, Q)$ where 1 is the identity map of $Q$ and let $\psi$ be a local diffeomorphism of $Q$ such that $\psi^{(l)}$ maps an open subset $W^{l}$ of a $\Gamma$-neighbourhood $V^{l}$ of $j_{z_{0}}^{l}(1)$ into $V^{l}$. We set $G^{l}=\left\{j_{z}^{l}(1) \in \tilde{J}^{l}(Q, Q) \mid z \in Q\right\}$. Let $\left\{y_{j}^{l}\right\}_{j=1}^{m_{l}}$ be a fundamental system of differential invariants of $\mathscr{L}_{\Gamma}$ on $V^{l}$. If $\left(\psi^{(l)}\right)^{*} y_{j}^{l}=y_{j}^{l}\left(1 \leqq j \leqq m_{l}\right)$ on $G^{l} \cap W^{l}$, then we get $\left(\psi^{(l)}\right)^{*} y_{j}^{l}=y_{j}^{l}\left(1 \leqq j \leqq m_{l}\right)$ on $W^{l}$.

Proof. For a local diffeomorphism $f$ of $Q$ such that $j_{z}^{l}(f) \in W^{l}$, we shall define a local diffeomorphism $f^{l}$ of a neighbourhood of $j_{z}^{l}(f)$ onto a neighbourhood of $j_{z}^{l}(1) \in V^{l}$ by $f^{l}\left(j_{z^{\prime}}^{l}(g)\right)=j_{f\left(z^{\prime}\right)}^{l}\left(g \circ f^{-1}\right)$. Then we have $\psi^{(l)} \circ f^{l}=f^{l} \circ \psi^{(l)}$ if the composition is well-defined, since we have $\psi^{(l)} \circ f^{l}\left(j_{z^{\prime}}^{l}(g)\right)=\psi^{(l)}\left(j_{f\left(z^{\prime}\right)}^{l}\left(g \circ f^{-1}\right)\right)=j_{f\left(z^{\prime}\right)}^{l}\left(\psi \circ g \circ f^{-1}\right)=f^{l}\left(j_{z^{\prime}}^{l}(\psi \circ g)\right)=f^{l} \circ \psi^{(l)}\left(j_{z^{\prime}}^{l}(g)\right)$. Therefore, if we set $\left(f^{l}\right)^{*} y_{j}^{l}=\bar{y}_{j}^{l}\left(1 \leqq j \leqq m_{l}\right)$, then $\left\{\bar{y}_{j}^{l}\right\}_{j=1}^{m_{l}}$ is also a fundamental system of differential invariants of $\mathscr{L}_{\Gamma}$ at $j_{z}^{l}(f)$. Moreover we have $\left(\left(\psi^{(l)}\right)^{*} \bar{y}_{j}\right)\left(j_{z}^{l}(f)\right)=\left(\left(\psi^{(l)}\right)^{*}\left(f^{l}\right)^{*} y_{j}^{l}\right)\left(j_{z}^{l}(f)\right)=\left(\left(f^{l}\right)^{*}\left(\psi^{(l)}\right)^{*} y_{j}^{l}\right)\left(j_{z}^{l}(f)\right)=\left(\left(\psi^{(l)}\right)^{*}\right.$ $\left.y_{j}^{l}\right)\left(j_{z}^{l}(1)\right)=y_{j}^{l}\left(j_{z}^{l}(1)\right)=\left(\left(f^{l}\right)^{*} y_{j}^{l}\right)\left(j_{z}^{l}(f)\right)=\bar{y}_{j}^{l}\left(j_{z}^{l}(f)\right)\left(1 \leqq j \leqq m_{l}\right)$. Since $\left\{\bar{y}_{j}^{l}\right\}_{j=1}^{m^{l}}$ is also a fundamental system of differential invariants of $\mathscr{L}_{\Gamma}$ at $j_{z}^{l}(f) \in W^{l}$, we have $\left(\psi^{(l)}\right)^{*} y_{j}^{l}=y_{j}^{l}$ on $W^{l}$. 
6. Theorem 6.1. Let $\Gamma$ be a pseudo-group on $Q$ which is complete at $(z, 1)$ and let $s$ be a map of maximal rank of a neighbourhood of $x_{0} \in N$ to a neighbourhood of $z \in Q$. Let $\bar{l}$ be the order of $\mathscr{L}_{\Gamma}$ at $\left(x_{0}, s\right)$. Then there exists a sequence of differential equations $\left\{(B)_{l}\right\}_{l \geqq i}$ at $p^{l}=j_{x_{0}}^{l}(s) \in \tilde{J}^{l}(N, Q)$ satisfying the following properties:

(1) $(B)_{l}$ is generated by functions $y_{j}^{l}-\lambda_{j}^{l}\left(1 \leqq j \leqq m_{l}\right)$ where $\left\{\lambda_{j}^{l}\right\}_{j=1}^{m_{l}}$ (resp. $\left\{y_{j}^{l}\right\}_{j=1}^{m_{l}}$ ) is a family of functions locally defined at $x_{0}$ (resp. a fundamental system of differential invariants of $\mathscr{L}_{\Gamma}$ at $\left.p^{l}\right)$.

(2) $(B)_{l}$ is a $\Gamma$-automorphic system and possesses $s$ as a solution for any $l \geqq \bar{l}$.

Proof. We set $\lambda_{j}^{l}(x)=y_{j}^{l}\left(j_{x}^{l}(s)\right)$. Let $f^{1}$ and $f^{2}$ be solutions of $(B)_{i}$. Then we may assume that they are defined on a neighbourhood $U$ of $x_{0}$. We have clearly $y_{j}^{l}\left(j_{x}^{l}\left(f^{1}\right)\right)=y_{j}^{l}\left(j_{x}^{l}\left(f^{2}\right)\right)$ for any $x \in U$. Let $U^{l}$ be a neighbourhood of $p^{l}$ such that $U^{l} / D_{\mathscr{L}_{\Gamma}}^{(l)}$ is a manifold and let $\pi^{l}$ be the projection of $U^{l}$ onto $U^{l} / D_{\mathscr{L}_{\Gamma}}^{(l)}$. Then $y_{j}^{l}\left(j_{x}^{l}\left(f^{1}\right)\right)=y_{j}^{l}\left(j_{x}^{l}\left(f^{2}\right)\right)\left(1 \leqq j \leqq m_{l}\right)$ imply that $\pi^{l}\left(j_{x}^{l}\left(f^{1}\right)\right)=\pi^{l}\left(j_{x}^{l}\left(f^{2}\right)\right)$. Let $\omega$ be any local diffeomorphism of $U$. Then, if we set $\omega^{l}\left(j_{x}^{l}(f)\right)=j_{\omega^{-1}(x)}^{l}(f \circ \omega), \omega^{l}$ is a local diffeomorphism of $\tilde{J}^{l}(N, Q)$ and for any local diffeomorphism $\phi$ of $Q$, we have $\phi^{(l)} \circ \omega^{l}$ $=\omega^{l} \circ \phi^{(l)}$ if the compositions are defined. In particular, we have the commutativity for any $\phi \in \Gamma$. Therefore we get $\pi^{l}\left(j^{l}\left(f^{1} \circ \omega\right)\right)=\pi^{l}\left(j^{l}\left(f^{2} \circ \omega\right)\right)$. Let $W \times \Lambda$ be the product structure of $U$ associated with $f^{1}$. Then $f^{1}$ is the natural projection of $W \times \Lambda \rightarrow W$. We set $U_{\lambda}=W \times\{\lambda\} \subset W \times \Lambda$. Then we may consider that $f^{2} \mid U_{\lambda}$ is a diffeomorphism for any $\lambda \in \Lambda$. We set $f_{\lambda}^{i}=f^{i} \mid U_{\lambda}$ and denote by $\pi_{\lambda}$ the natural projection of $U$ onto $U_{\lambda}$. Then we have clearly $\pi_{\lambda}=\left(f_{\lambda}^{1}\right)^{-1} \circ f^{1}$. We set $g_{\lambda}=\left(f_{\lambda}^{2}\right) \circ\left(f_{\lambda}^{1}\right)^{-1}$. Then we have

$$
\begin{aligned}
g_{\lambda}^{(l)} \cdot j^{l}\left(f^{1} \circ \omega\right) & =j^{l}\left(\left(f_{\lambda}^{2}\right) \circ\left(f_{\lambda}^{1}\right)^{-1}\left(f^{1} \circ \omega\right)\right) \\
& =j^{l}\left(\left(f_{\lambda}^{2} \circ \pi_{\lambda^{\circ}} \omega\right)=j^{l}\left(f^{2} \circ \pi_{\lambda^{\circ}} \circ \omega\right) .\right.
\end{aligned}
$$

On the other hand, if we define a local map $\pi_{\lambda}^{l}$ of $J^{l}(N, Q)$ into $J^{l}(N, Q)$ by $\pi_{\lambda}^{l}\left(j_{x}^{l}(f)\right)=j_{x}^{l}\left(f \circ \pi_{\lambda}\right)$, then by the same argument as for the map $\omega^{l}$, we have $\pi_{\lambda}^{l} \circ \phi^{(l)}=\phi^{(l)} \circ \pi_{\lambda}^{l}$ for any local diffeomorphism $\phi$ of $Q$. Therefore $\pi^{l}\left(j^{l}\left(f^{1} \circ \pi_{\lambda}\right)\right)=\pi^{l}\left(j^{l}\left(f^{2} \circ \pi_{\lambda}\right)\right)$ and we get also $\pi^{l}\left(j^{l}\left(f^{1} \circ \pi_{\left.\lambda^{\circ} \circ \omega\right)}\right)=\pi^{l}\left(j^{l}\left(f^{2} \circ\right.\right.\right.$ $\left.\left.\pi_{\lambda^{\circ}} \omega\right)\right)$. Since we have $\pi^{l}\left(j^{l}\left(f^{1} \circ \pi_{\lambda^{\circ}} \omega\right)\right)=\pi^{l}\left(j^{l}\left(f^{1} \circ \omega\right)\right)$, we get 


$$
\pi^{l}\left(g_{\lambda}^{(l)} j^{l}\left(f^{1} \circ \omega\right)=\pi^{l}\left(j^{l}\left(f^{1} \circ \omega\right)\right)\right.
$$

That is, we have

$$
\left(g_{\lambda}^{(l)}\right)^{*} y_{j}^{l}\left(j^{l}\left(f^{1} \circ \omega\right)\right)=y_{j}^{l}\left(j^{l}\left(f^{1} \circ \omega\right)\right) \quad\left(1 \leqq j \leqq m_{l}\right)
$$

Since $\omega$ is any local diffeomorphism of $U$, we have $\left(g_{\lambda}^{(l)}\right)^{*} \cdot y_{j}^{l}=y_{j}^{l}(1 \leqq j$ $\left.\leqq m_{l}\right)$.

If $l \geqq l, f^{1}$ and $f^{2}$ are solutions of all $(B)_{k}, k \geqq 0$ by Lemma 4.1. Therefore $g_{\lambda}^{(l)} \cdot y_{j}^{l}=y_{j}^{l}\left(1 \leqq j \leqq m_{l}, l=0,1,2, \ldots\right)$. Since $\Gamma$ is complete at $(z, 1)$, by Lemma 5.1, we get $g_{\lambda} \in \Gamma$ for any $\lambda \in \Lambda$. We have $f^{2}(w, \lambda)$ $=g_{\lambda} \circ f^{1}(w, \lambda)$ for any $(w, \lambda) \in W \times \Lambda=U$.

Now let $\left\{\lambda_{1}, \ldots, \lambda_{t}\right\}$ be a coordinate on $\Lambda$ and we shall consider the system of partial differential equations

$$
\left(B^{\prime}\right)_{l}: \begin{cases}y_{j}^{l}\left(j_{x}^{l}(f)\right)=\lambda_{j}^{l}(x) & \left(1 \leqq j \leqq m_{l}\right), \\ \frac{\partial z_{k}\left(f_{\lambda^{\circ}}\left(s_{\lambda}\right)^{-1}\right)}{\partial \lambda_{i}}=0 & (1 \leqq i \leqq t, 1 \leqq k \leqq q)\end{cases}
$$

where $\left\{z_{1}, \ldots, z_{q}\right\}$ is a coordinate system on $Q$. Let $f^{1}, f^{2}$ be solutions of $\left(B^{\prime}\right)_{l}$. Then if $l \geqq \bar{l}$, we have $f^{2}(w, \lambda)=g_{\lambda^{\circ}} f^{1}(w, \lambda), g_{\lambda} \in \Gamma$. On the other hand, $g_{\lambda}=f_{\lambda}^{2} \circ\left(f_{\lambda}^{1}\right)^{-1}=\left\{f_{\lambda}^{2} \circ\left(s_{\lambda}\right)^{-1}\right\} \circ\left\{f_{\lambda}^{1} \circ\left(s_{\lambda}\right)^{-1}\right\}^{-1}$. Since $f_{\lambda}^{2} \circ\left(s_{\lambda}\right)^{-1}$ and $f_{\lambda}^{1} \circ\left(s_{\lambda}\right)^{-1}$ are independent of $\lambda, g_{\lambda}$ is also independent of $\lambda$. Therefore we get $f^{2}=g \circ f^{1}, g \in \Gamma$. This implies that $\left(B^{\prime}\right)_{l}$ is $\Gamma$-automorphic if $l \geqq l$. If we denote by $\mathscr{I}_{p}^{l}$ the maximal integral manifold of $D_{\mathscr{L}_{\Gamma}}^{(l)}$, we have $I(B)_{l}=\bigcup_{p \in s(l)} \mathscr{I}_{p}^{l} \cap U^{l}$. On the other hand, since $\left(B^{\prime}\right)_{l}$ is $\Gamma$-automorphic, we have also $I\left(B^{\prime}\right)_{l}=\bigcup_{p \in s(l)} \mathscr{I}_{p}^{l} \cap U^{l}$. This implies that $(B)_{l}$ is also a $\Gamma$-automorphic system. This completes the proof of Theorem 6.1 .

\section{§3. Equivalence Problem of Automorphic Systems}

7. Definition 7.1. Let $(A)^{l}$ be a system of differential equations at $j_{x_{0}}^{l}(f) \in \tilde{J}^{l}(N, Q)$ and let $\Gamma$ be a pseudo-group on $Q . \quad(A)^{l}$ is said to admit a pseudo-group $\Gamma$ if, for each solution $s$ of $(A)^{l}$ and any element $\phi \in \Gamma$ which is defined on a neighbourhood of $s\left(x_{0}\right), \phi \circ s$ is also a solution of $(A)^{l}$. 
Let $\Gamma$ be a pseudo-group on $Q$ which is complete at $(z, 1)$. Let $s$ be a map of maximal rank of a neighbourhood of $x_{0}$ to $Q$ with $s\left(x_{0}\right)$ $=z$ and let $l_{0}$ be the order of $\mathscr{L}_{\Gamma}$ at $\left(x_{0}, s\right)$. For a fundamental system of differential invariants $\left\{y_{j}^{l}\right\}_{j=1}^{m_{1}}$ of $\mathscr{L}_{\Gamma}$ at $j_{x_{0}}^{l}(s) \in \tilde{J}^{l}(N, Q)$, we set $\lambda_{j}^{l}(x)$ $=y_{j}^{l}\left(j_{x}^{l}(s)\right)$. Then, by Th. 6.1 , the system of differential equations $(B)_{\lambda}^{l}$ : $y_{j}^{l}=\lambda_{j}^{l}\left(1 \leqq j \leqq m_{l}\right)$ is a $\Gamma$-automorphic system for $l \geqq l_{0}$.

Lemma 7.1. The maximal pseudo-group which is complete at $(z, 1)$ and which is admitted by the system of differential equations $(B)_{\lambda}^{l}$ is equal to $\Gamma$ on a neighbourhood of $z$.

Proof. Let $\tilde{\Gamma}$ be the maximal pseudo-group which is complete at $(z, 1)$ and which $(B)_{\lambda}^{l}$ admits. Then $\tilde{\Gamma}$ is a pseudo-group on a neighbourhood $\mathscr{U}$ of $z$ and clearly we have $\tilde{\Gamma} \supset \Gamma \mid \mathcal{U}$. Let $\left\{\tilde{y}_{j}^{l}\right\}_{j=1}^{\tilde{m}_{l}}$ be a fundamental system of differential invariants of $\mathscr{L}_{\tilde{\Gamma}}$ at $j_{x_{0}}^{l}(s)$. Then we have $\tilde{m}_{l} \leqq m_{l}$ and we may assume that $\tilde{y}_{j}^{l}=y_{j}^{l}\left(1 \leqq j \leqq \tilde{m}_{l}\right)$. We shall show that $\Gamma$ (resp. $\tilde{\Gamma}$ ) is locally defined at $j_{x_{0}}^{l}(s)$ by $\left\{y_{j}^{l}\right\}_{j=1}^{m_{l}}$ (resp. $\left\{\tilde{y}_{j}^{l}\right\}_{j=1}^{\tilde{m}_{l}}$ ) for $l \geqq \max \left(l_{0}, \tilde{l}_{0}\right)$ where $l_{0}$ (resp. $\left.\tilde{l}_{0}\right)$ is the order of $\mathscr{L}_{\tilde{\Gamma}}\left(\right.$ resp. $\mathscr{L}_{\tilde{\Gamma}}$ ) at $\left(x_{0}, s\right)$. Let $\phi$ be a local diffeomorphism of $Q$ such that $\left(\phi^{(l)}\right)^{*} y_{j}^{l}=y_{j}^{l}$ $\left(1 \leqq j \leqq m_{l}\right)$. Then if $f$ is a solution of $(B)_{\lambda}^{l}$, so is $\phi \circ f$. We set $\bar{f}=\phi \circ f$. Since $f$ and $\bar{f}$ are solutions of $(B)_{\lambda}^{l}$, as we proved in Th. 6.1, $g_{\lambda}=\left(\bar{f}_{\lambda}\right)$ 。 $\left(f_{\lambda}\right)^{-1}$ is an element of $\Gamma$ and $g_{\lambda}$ is independent of $\lambda$ which we denote by $g$. Then we get $\phi=g \in \Gamma$. Clearly any element $\psi$ of $\Gamma$ satisfies $\left(\psi^{(l)}\right)^{*} y_{j}^{l}=y_{j}^{l}\left(1 \leqq j \leqq m_{l}\right)$. This implies that $\Gamma$ is locally defined at $z=s\left(x_{0}\right)$ by the system of equations

$$
\left(\phi^{(l)}\right)^{*} y_{j}^{l}=y_{j}^{l} \quad\left(1 \leqq j \leqq m_{l}\right)
$$

Similarly $\tilde{\Gamma}$ is locally defined at $z$ by the system of equations

$$
\left(\phi^{(l)}\right)^{*} \tilde{y}_{j}^{l}=\tilde{y}_{j}^{l} \quad\left(1 \leqq j \leqq \tilde{m}_{l}\right)
$$

Therefore we have only to see that $m_{l}=\tilde{m}_{l}$ for $l \geqq \max \left(l_{0}, \tilde{l}_{0}\right)$. Now let $D^{l}$ (resp. $\tilde{D}^{l}$ ) be the involutive distribution on $\tilde{J}^{l}(N, \mathscr{U})$ induced from $\Gamma$ (resp. $\tilde{\Gamma}$ ) and let $\mathscr{I}_{p}^{l}$ (resp. $\tilde{I}_{p}^{l}$ ) be the maximal integral manifold of $D^{l}$ (resp. $\left.\widetilde{D}^{l}\right)$ through $p \in \tilde{J}^{l}(N, \mathscr{U})$. Then, as we proved in Lemma 4.1, we have $I(B)_{\lambda}^{l} \cap \mathscr{U}^{l}=\bigcup_{p \in s(l)} \mathscr{I}_{p}^{l} \cap \mathscr{U}^{l}$ and $I(\widetilde{B})_{\lambda}^{l} \cap \mathscr{U}^{l}=\underset{p \in s(l)}{\cup} \tilde{\mathscr{I}}_{p}^{l} \cap \mathscr{U}^{l}$ for 
a neighbourhood $\mathscr{U}^{l}$ of $j_{x_{0}}^{l}(s)$. Since $\tilde{\Gamma} \supset \Gamma \mid \mathscr{U}$, we get $\underset{p \in s(l)}{\cup} \tilde{\mathscr{I}}_{p}^{l} \cap \mathscr{U}^{l} \supset \underset{p \in s(l)}{\cup}$ $\mathscr{I}_{p}^{l} \cap \mathscr{U}^{l}$. On the other hand, since $(B)_{\lambda}^{l}$ admits $\tilde{\Gamma}$, we have $\underset{p \in s(l)}{\cup} \mathscr{I}_{p}^{l} \cap \mathscr{U}^{l}$ $=I(B)_{\lambda}^{l} \cap \mathscr{U}^{l} \supset \underset{p \in s(l)}{\cup} \tilde{\mathscr{I}}_{p}^{l} \cap \mathscr{U}^{l}$. Therefore we get $\underset{p \in s(l)}{\cup} \mathscr{I}_{p}^{l} \cap \mathscr{U}^{l}=\bigcup_{p \in s(l)}^{\bigcup} \tilde{\mathscr{I}}_{p}^{l} \cap \mathscr{U}^{l}$, that is, $I(B)_{\lambda}^{l} \cap \mathscr{U}^{l}=I(\widetilde{B})_{\lambda}^{l} \cap \mathscr{U}^{l}$. Thus we get $m_{l}=\tilde{m}_{l}$. This completes the proof of Lemma 7.1 .

Lemma 7.2. Let $\Gamma$ be a pseudo-group on $Q$ which is complete at $(z, 1)$ for any $z \in Q$. Let $\phi$ be an isomorphism of the $\Gamma$-automorphic system $(B)_{\lambda}^{l}: y_{j}^{l}=\lambda_{j}^{l}\left(1 \leqq j \leqq m_{l}\right)$ to the $\Gamma$-automorphic system $(B)_{\mu}^{l}: y_{j}^{l}=\mu_{j}^{l}$ $\left(1 \leqq j \leqq m_{l}\right)$. Then $\phi$ is an element of the normalizer $\mathcal{N}(\Gamma)$ of $\Gamma$ in $\Gamma_{Q}$ which is the pseudo-group of all local diffeomorphisms of $Q$.

Proof. $\phi$ is a diffeomorphism of a neighbourhood $\mathscr{U}$ of $z$ onto a neighbourhood $\mathscr{U}^{\prime}$ of $z^{\prime}=\phi(z)$. We set $\Gamma^{\prime}=\phi \cdot(\Gamma \mid \mathscr{U}) \cdot \phi^{-1}$. Then $\Gamma^{\prime}$ is a pseudo-group on $\mathscr{U}^{\prime}$ and $\Gamma^{\prime}$ is complete at $\left(z^{\prime}, 1\right)$. Since $\phi$ is an isomorphism of $(B)_{\lambda}^{l}$ to $(B)_{\mu}^{l}$, for any solution $s_{2}$ of $(B)_{\mu}^{l}, \phi^{-1} \circ s_{2}$ is a solution of $(B)_{\lambda}^{l}$ and $g \circ\left(\phi^{-1} \circ S_{2}\right), g \in \Gamma$, is also a solution of $(B)_{\lambda}^{l}$. Therefore $\left(\phi \cdot g \cdot \phi^{-1}\right) \circ s_{2}$ is a solution of $(B)_{\mu}^{l}$, that is, $(B)_{\mu}^{l}$ admits $\Gamma^{\prime}$. By Lemma 7.1 , we have $\Gamma \mid \mathscr{U}^{\prime} \supset \Gamma^{\prime}$. If $\Gamma\left|\mathscr{U}^{\prime} \neq \Gamma^{\prime}, \phi^{-1} \cdot\left(\Gamma \mid \mathscr{U}^{\prime}\right) \cdot \phi \supsetneqq \Gamma\right| \mathscr{U}$ and $(B)_{\lambda}^{l}$ admits $\phi^{-1} \cdot\left(\Gamma \mid \mathscr{U}^{\prime}\right) \cdot \phi$. This contradicts to the maximality of $\Gamma \mid \mathscr{U}$. Therefore we get $\Gamma^{\prime}=\Gamma \mid \mathscr{U}^{\prime}$. This implies that $\phi \in \mathcal{N}(\Gamma)$.

For a fundamental system of differential invariants $\left\{y_{j}^{l}\right\}_{j=1}^{m_{l}}$ of $\mathscr{L}_{\Gamma}$ at $j_{x_{0}}^{l}(s) \in \tilde{J}^{l}(N, Q)$, we have a neighbourhood $\mathscr{W}$ of $\left(y_{1}^{l}\left(j_{x_{0}}^{l}(s)\right), \ldots, y_{m_{l}}^{l}\right.$ $\left.\left(j_{x_{0}}^{l}(s)\right)\right) \in \boldsymbol{R}^{m_{l}}$ and a $\Gamma$-neighbourhood $\mathscr{U}^{l}$ of $j_{x_{0}}^{l}(s)$ such that $\mathscr{U}^{l}$ possesses a product structure $\mathscr{W} \times \mathscr{V}$ and such that the natural projection of $\mathscr{W} \times \mathscr{V}$ onto $\mathscr{W}$ is equal to $\left(y_{1}^{l}, \ldots, y_{m_{l}}^{l}\right)$. For $\phi \in \mathscr{N}(\Gamma)$, there are analytic functions $\xi_{1}, \ldots, \xi_{m_{l}}$ defined on a neighbourhood of $\left(y_{1}^{l}\left(j_{x_{0}}^{l}(s)\right), \ldots, y_{m_{l}}^{l}\left(j_{x_{0}}^{l}(s)\right)\right)$ $\in \boldsymbol{R}^{m_{l}}$ such that $\phi^{(l) * y_{j}^{l}}=\xi_{j}\left(y_{1}^{l}, \ldots, y_{m_{l}}^{l}\right)\left(1 \leqq j \leqq m_{l}\right)$ and such that $\xi=\left(\xi_{1}, \ldots\right.$, $\left.\xi_{m_{l}}\right)$ is a local diffeomorphism of $\mathscr{W}$. We set $D(\phi)=\xi$ and $y^{l}=\left(y_{1}^{l}, \ldots\right.$,

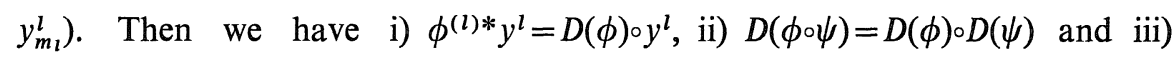
$D\left(\phi^{-1}\right)=D(\phi)^{-1}$. Thus $\mathscr{N}(\Gamma)$ induces a pseudo-group $D(\mathscr{N}(\Gamma))$ on $\mathscr{W}$.

Theorem 7.1. Let $\Gamma$ be a pseudo-group on $Q$ which is complete at $(z, 1)$ for any $z \in Q$. Let $\left\{y_{j}^{l}\right\}_{j=1}^{m_{l}}$ be a fundamental system of differential invariants of $\mathscr{L}_{\Gamma}$ at $j_{x_{0}}^{l}(s) \in \tilde{J}^{l}(N, Q)$ and let $l_{0}$ be the order of 
$\mathscr{L}_{\Gamma}$ at $\left(x_{0}, s\right)$. For $l \geqq l_{0}$, a $\quad \Gamma$-automorphic system $y_{j}^{l}=\lambda_{j}^{l}\left(1 \leqq j \leqq m_{l}\right)$ is isomorphic to another $\Gamma$-automorphic system $y_{j}^{l}=\mu_{j}^{l}\left(1 \leqq j \leqq m_{l}\right)$ if and only if there exists an element $D(\phi) \in D(\mathscr{N}(\Gamma))$ such that $\mu^{l}=D(\phi) \circ \lambda^{l}$ where $\mu^{l}$ (resp. $\lambda^{l}$ ) is a local map of a neighbourhood of $x_{0}$ to $\mathbb{R}^{m_{l}}$ defined by $\mu^{l}(x)=\left(\mu_{1}^{l}(x), \ldots, \mu_{m_{l}}^{l}(x)\right)$ (resp. $\lambda^{l}(x)=\left(\lambda_{1}^{l}(x), \ldots, \lambda_{m_{l}}^{l}(x)\right)$.

Proof. Let $\psi$ be an isomorphism of $y_{j}^{l}=\lambda_{j}^{l}\left(1 \leqq j \leqq m_{l}\right)$ to $y_{j}^{l}=\mu_{j}^{l}$ $\left(1 \leqq j \leqq m_{l}\right)$. Then by Lemma 7.2, $\psi$ must be an element of $\mathscr{N}(\Gamma)$. We set ' $y_{j}^{l}=\psi^{*} y_{j}^{l}\left(1 \leqq j \leqq m_{l}\right)$. Then the solution space of $y_{j}^{l}=\lambda_{j}^{l}\left(1 \leqq j \leqq m_{l}\right)$ is equal to the solution space of ' $y_{j}^{l}=\mu_{j}^{l}\left(1 \leqq j \leqq m_{l}\right)$. If we set ' $y^{l}=\left({ }^{\prime} y_{1}^{l}\right.$, $\left.\ldots,{ }^{\prime} y_{m_{l}}^{l}\right)$ (resp. $\left.y^{l}=\left(y_{1}^{l}, \ldots, y_{m_{l}}^{l}\right)\right)$, we have an element $D(\psi) \in D(\mathscr{N}(\Gamma))$ such that ' $y^{l}=D(\psi) \circ y^{l}$. Then the system ' $y^{l}=\mu^{l}$ is expressed by $D(\psi) \circ y^{l}=\mu^{l}$, that is, $y^{l}=D\left(\psi^{-1}\right) \circ \mu^{l}$. This implies that the system of differential equations $y^{l}=D\left(\psi^{-1}\right) \circ \mu^{l}$ is equal to the system of differential equations $y^{l}$ $=\lambda^{l}$. Therefore we get $D(\psi) \circ \lambda^{l}=\mu^{l}$. Conversely we assume that there is an element $D(\phi) \in D(\mathscr{N}(\Gamma))$ such that $\mu^{l}=D(\phi) \circ \lambda^{l}$. Then we have $y^{l}\left(j_{x}^{l}\left(s_{2}\right)\right)=\mu^{l}(x)=D(\phi) \circ \lambda^{l}(x)=D(\phi) \circ y^{l}\left(j_{x}^{l}\left(s_{1}\right)\right)=y^{l}\left(j_{x}^{l}\left(\phi \circ s_{1}\right)\right)$. Since $y^{l}=\mu^{l}$ is $\Gamma$-automorphic, we have an element $g \in \Gamma$ such that $\phi \circ s_{1}=g \circ s_{2}$. Since $\phi \in \mathscr{N}(\Gamma)$ and $s_{2}^{\prime}=g \circ s_{2}$ is a solution of $y^{l}=\mu^{l}$ and since the system $y^{l}=\lambda^{l}$ is also $\Gamma$-automorphic, it is easy to see that $\phi$ is an isomorphism of $y^{l}=\lambda^{l}$ to $y^{l}=\mu^{l}$. This completes the proof of Theorem 7.1.

\section{§4. A Remark on Completeness of Pseudo-groups}

8. Definition 8.1. Let $\Gamma$ be a pseudo-group on $Q$. Let $(A)$ be a system of differential equations at $j_{z}^{l}(1) \in \tilde{J}^{l}(Q, Q) . \quad \Gamma$ is said to be locally defined at $z$ by $(A)$ if the following condition is satisfied: Let $\phi$ be a local diffeomorphism of $Q$ such that $\phi^{(k)}$ maps an open subset $W^{k} \ni j_{z \prime}^{k}\left(f^{\prime}\right)$ of a certain $\Gamma$-neighbourhood $V^{k}$ of $j_{z}^{k}(1)$ into $V^{k}$ for any $k \geqq 0$. Then ' $\phi \in \Gamma$ if and only if ' $\phi$ is a solution of $(A)$.

Proposition 8.1. Let $\Gamma$ be a pseudo-group on $Q$ which is complete at $(z, 1)$. Then $\Gamma$ is locally defined at $z$ by a system of differential equations.

Proof. Let $p=j_{z}^{l}(1) \in \tilde{J}^{l}(Q, Q)$. By Lemma 4.1, we can define the 
order of $\Gamma$ at $(z, 1)$. We assume that $l$ is the order of $\Gamma$ at $(z, 1)$. In the proof of Lemma 7.1, we showed that, if $\left\{y_{j}^{l}\right\}_{j=1}^{m_{l}}$ is a fundamental system of differential invariants of $\Gamma$ at $j_{z}^{l}(1) \in \tilde{J}^{l}(Q, Q), \Gamma$ is locally defined at $z$ by $\phi^{(l) * y_{j}^{l}}=y_{j}^{l}\left(1 \leqq j \leqq m_{l}\right)$. We set $y_{j}^{l}\left(j_{z}^{l}(1)\right)=\lambda_{j}^{l}(z)$. Then, by Lemma 5.2, $\Gamma$ is locally defined at $z$ by $y_{j}^{l}\left(j_{z}^{l}(\phi)\right)=\lambda_{j}^{l}(z)\left(1 \leqq j \leqq m_{l}\right)$. This system is a system of differential equations at $j_{z}^{l}(1) \in \tilde{J}^{l}(Q, Q)$ with respect to the unknown map $\phi$.

Proposition 8.2. Let $\Gamma$ be a pseudo-group on $Q$ such that $\mathscr{L}_{\Gamma}$ is a regular Lie algebra sheaf and such that $\Gamma$ is locally defined at $z_{0} \in Q$ by a system of differential equations $(A)^{l}$ at $j_{z_{0}}^{l}(1) \in \tilde{J}^{l}(Q, Q)$. Then $\Gamma$ is complete at $\left(z_{0}, 1\right)$.

Proof. Let $\left\{y_{j}^{k}\right\}_{j=1}^{m_{k}}$ be a fundamental system of differential invariants of $\mathscr{L}_{\Gamma}$ at $j_{z_{0}}^{k}(1)$. We set $\lambda_{j}^{k}(z)=y_{j}^{k}\left(j_{z}^{k}(1)\right)$. We denote by $(B)^{k}$ the system of differential equations generated by $y_{j}^{k}-\lambda_{j}^{k}\left(1 \leqq j \leqq m_{k}\right)$. Then, there is a neighbourhood $\mathscr{U}^{l}$ of $j_{z_{0}}^{l}(1)$ such that $I(A)^{l} \cap \mathscr{U}^{l} \supset I(B)^{l} \cap \mathscr{U}^{l}$, where $I(A)^{l}$ (resp. $\left.I(B)^{l}\right)$ is the set of integral points of $(A)^{l}$ (resp. $(B)^{l}$ ). Since clearly $(A)^{l}$ is $\Gamma$-automorphic, $(B)^{l}$ is also $\Gamma$-automorphic. Let $k$ be any integer such that $k \geqq l$. Then $p^{k-l}(A)^{l}$, the $(k-l)$-th prolongation of $(A)^{l}$ to a neighbourhood of $j_{z_{0}}^{k}(1) \in \tilde{J}^{k}(Q, Q)$, possesses the same solution space as $(A)^{l}$ and therefore $\Gamma$-automorphic. Since we have a neighbourhood $\mathscr{U}^{k}$ of $j_{z_{0}}^{k}(1)$ such that $I\left(p^{k-l}(A)^{l}\right) \cap \mathscr{U}^{k} \supset I(B)^{k} \cap \mathscr{U}^{k},(B)^{k}$ is also $\Gamma$-automorphic. Let $\varphi$ be any local diffeomorphism of $Q$ such that $\varphi$ maps an open subset $W^{k} \ni j_{z}^{k}(f)$ of a $\Gamma$-neighbourhood $V^{k}$ of $j_{z_{0}}^{k}(1)$ into $V^{k}$ and $\left(\varphi^{(k)}\right)^{*} y_{j}^{k}=y_{j}^{k}\left(1 \leqq j \leqq m_{k}\right)$ on ' $W^{k}$ for any $k \geqq 0$. By Lemma 5.2, this last condition is equivalent to $y_{j}^{k}\left(j_{z}^{k}(\varphi)\right)=\lambda_{j}^{k}(z)\left(1 \leqq j \leqq m_{k}\right)$ on $G^{k}$ $\cap^{\prime} W^{k}$. Therefore ' $\varphi$ is a solution of any $(B)^{k}(k \geqq 0)$ and, in particular, ${ }^{\prime} \varphi$ is a solution of $(A)^{l}$, that is, ' $\varphi \in \Gamma$. Conversely let $\varphi$ be any local diffeomorphism of $Q$ such that $\varphi$ maps an open subset $W^{k} \ni j_{z}^{k}(f)$ of a $\Gamma$-neighbourhood $V^{k}$ of $j_{z_{0}}^{k}(1)$ into $V^{k}$ and ' $\varphi \in \Gamma$. Then ' $\varphi$ is a solution of $(A)^{l}$ and therefore of $(B)^{l}$. Since ' $\varphi$ is also a solution of $p^{k-l}(A)^{l}$ for any $k \geqq l$, it is also a solution of $(B)^{k}(k \geqq l)$ and therefore a solution of $(B)^{k}(k \geqq 0)$. ' $\varphi$ is a solution of $(B)^{k}$ if and only if $\left(\varphi^{(k)}\right)^{*} y_{j}^{k}=y_{j}^{k}$ $\left(1 \leqq j \leqq m_{k}\right)$ on ' $W^{k}$. This implies that $\left(\varphi^{(k)}\right)^{*} y_{j}^{k}=y_{j}^{k}\left(1 \leqq j \leqq m_{k}\right)$ on ' $W^{k}$ for $k \geqq 0$. 
Remark 8.1. Let $\Gamma$ be a pseudo-group on $Q$ which is complete at $(z, 1)$. Then $\Gamma$ is complete at any $(x, f)$ with $f(x)=z$.

This is the particular case of Lemma 5.1. Therefore $\Gamma$ may be said to be complete at $z$ if it is complete at $(z, 1)$. It is now clear that, if $\Gamma$ is complete at $(z, 1)$ for any $(z, 1)$, it is complete at any $(z, f)$. Therefore $\Gamma$ may be said to be complete if it is complete at $(z, 1)$ for any $z \in Q$.

\section{§5. Examples}

9. Example 1. We set $I_{0}=(-a, 0) \cup(0, a) \subset \mathbb{R}$ and consider the system of differential equations

$$
\left\{\begin{array}{l}
\frac{\partial u}{\partial x}=\alpha(x, y) \cdot u \\
\frac{\partial u}{\partial y}=\beta(x, y) \cdot u
\end{array}\right.
$$

at $j_{z_{0}}^{1}(s) \in \tilde{J}^{1}\left(\boldsymbol{R}^{2}, I_{0}\right), z_{0}=\left(x_{0}, y_{0}\right)$. Let $\mathscr{L}$ be a Lie algebra sheaf on $I_{0}$ generated by $u \cdot \frac{\partial}{\partial u}$. Then, we can easily see that $\left\{x, y, \frac{\partial u}{\partial x} / u, \frac{\partial u}{\partial y} / u\right\}$ is a fundamental system of differential invariants of $\mathscr{L}$ at any $p \in \tilde{J}^{1}\left(\boldsymbol{R}^{2}\right.$, $I_{0}$ ) and that $\mathscr{L}$ is determined by the system, that is, the order of $\mathscr{L}$ at $(\alpha(p), f)$ is 1 for any $f$. We denote by $\Gamma$ the pseudo-group on $\mathbb{I}_{0}$ determined by the system $\left\{x, y, \frac{\partial u}{\partial x} / u, \frac{\partial u}{\partial y} / u\right\}$. Then $\Gamma$ is complete at any $(z, 1), z \in I_{0}$. By Theorem 6.1, the system of differential equations

$$
\left\{\begin{array}{l}
\frac{\partial u}{\partial x} / u=\alpha(x, y) \\
\frac{\partial u}{\partial y} / u=\beta(x, y)
\end{array}\right.
$$

is $\Gamma$-automorphic if it possesses a solution, that is, (*) is $\Gamma$-automorphic. The integrability condition of $(*)$ is given by $\frac{\partial \alpha}{\partial y}=\frac{\partial \beta}{\partial x}$.

Now for any $\phi \in \mathscr{N}(\Gamma)$, we have $\left(\phi^{(1)}\right)^{*}\left(\frac{\partial u}{\partial x} / u\right)=F\left(x, y, \frac{\partial u}{\partial x} / u\right.$, 
$\left.\frac{\partial u}{\partial y} / u\right)$. On the other hand, $\left(\phi^{(1)}\right)^{*}\left(\frac{\partial u}{\partial x} / u\right)=\frac{\partial \phi(u)}{\partial x} / \phi(u)=\phi^{\prime}(u) \cdot \frac{\partial u}{\partial x} /$ $\phi(u)=\left(\frac{\phi^{\prime}(u)}{\phi(u)} \cdot u\right) \cdot \frac{\partial u}{\partial x} / u$. Therefore we get $\frac{\phi^{\prime}(u)}{\phi(u)} \cdot u=$ constant $=c$. Similarly, for $\phi \in \mathscr{N}(\Gamma),\left(\phi^{(1)}\right) *\left(\frac{\partial u}{\partial y} / u\right)=\left(\frac{\phi^{\prime}(u)}{\phi(u)} \cdot u\right) \cdot \frac{\partial u}{\partial y} / u=c \cdot \frac{\partial u}{\partial y} / u$. Therefore if

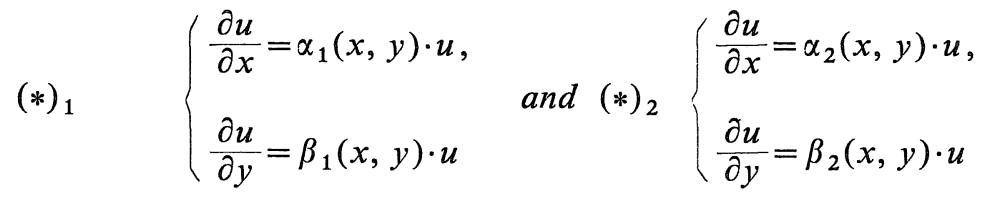

are isomorphic, then there is a constant $c$ such that $\alpha_{2}=c \cdot \alpha_{1}$ and $\beta_{2}=c \cdot \beta_{1}$. Conversely if $\alpha_{2}=c \cdot \alpha_{1}$ and $\beta_{2}=c \cdot \beta_{1}$ where $|c-1|$ is a suffciently small number, we can prove that $(*)_{1}$ and $(*)_{2}$ are isomorphic as the following way: Let $\mathscr{A}\left(I_{0}\right)$ be the Lie algebra sheaf on $I_{0}$ generated by all local vector fields on $I_{0}$. We denote by $\mathscr{N}(\mathscr{L})$ the normalizer of $\mathscr{L}$ in $\mathscr{A}\left(I_{0}\right)$. Then we can easily see that $\mathscr{N}(\mathscr{L})$ is the Lie algebra sheaf generated by $(u \cdot \log (u)) \cdot \frac{\partial}{\partial u}$ and $u \cdot \frac{\partial}{\partial u}$. Let $\phi_{t}\left(\right.$ resp. $\left.\psi_{t}\right)$ be a local 1-parameter group of local transformations induced from $(a \cdot u$. $\log (u)+b \cdot u) \cdot \frac{\partial}{\partial u}\left(\operatorname{resp} .(a \cdot u \cdot \log (u)) \cdot \frac{\partial}{\partial u}\right)$. Then we get $D\left(\phi_{t}\right)=D\left(\psi_{t}\right)$. On the other hand it is easy to see that $\psi_{t}(u)=u^{e^{a \cdot t}}$ and we have

$$
\begin{aligned}
\left(\psi_{t}^{(1)}\right) * \frac{\partial u}{\partial x} / u=\frac{\partial\left(u^{e^{a \cdot t}}\right)}{\partial x} / u^{e^{a \cdot t}} & =e^{a \cdot t} \cdot u^{e^{a \cdot t}-1} \cdot \frac{\partial u}{\partial x} / u^{e^{a \cdot t}} \\
& =e^{a \cdot t} \cdot \frac{\partial u}{\partial x} / u
\end{aligned}
$$

and

$$
\left(\psi_{t}^{(1)}\right) * \frac{\partial u}{\partial y} / u=e^{a \cdot t} \cdot \frac{\partial u}{\partial y} / u
$$

This implies that, for a positive number $c$ such that $|c-1|$ is sufficiently small, there exists an element $\phi \in \Gamma$ such that $D(\phi) \circ(\alpha, \beta)=(c \cdot \alpha, c \cdot \beta)$. Therefore, by Theorem $7.1,(*)_{1}$ and $(*)_{2}$ are isomorphic.

Example 2. We set $\boldsymbol{R}_{*}^{2}=\left\{(x, y) \mid(x, y) \in \mathbb{R}^{2}, y \neq 0\right\}$ and let us consider the system of differential equations 
(*)

$$
\left\{\begin{array}{l}
y \cdot \frac{\partial x}{\partial u}=\alpha(u, v) \\
y \cdot \frac{\partial x}{\partial v}=\beta(u, v)
\end{array}\right.
$$

at $j_{w_{0}}^{1}(s) \in \tilde{J}^{1}\left(\boldsymbol{R}^{2}, \boldsymbol{R}_{*}^{2}\right), w_{0}=\left(u_{0}, v_{0}\right)$. Let $\mathscr{L}$ be a Lie algebra sheaf on $\mathbb{R}_{*}^{2}$ generated by various local vector fields of the form $\xi(x) \cdot \frac{\partial}{\partial x}-\xi^{\prime}(x)$. $y \cdot \frac{\partial}{\partial y}$ where $\xi(x)$ is any local analytic function. Then $\mathscr{L}$ is of infinite type and we can easily see that $\operatorname{dim} D^{(1)}=3$ and that $\left\{u, v, y \cdot \frac{\partial x}{\partial u}, y\right.$. $\left.\frac{\partial x}{\partial v}, \frac{D(x, y)}{D(u, v)}\right\}$ is a fundamental system of differential invariants of $\mathscr{L}$ at any $p \in \tilde{J}^{1}\left(\boldsymbol{R}^{2}, \mathbb{R}_{*}^{2}\right)$ and conversely $\mathscr{L}$ is determined by the system. Therefore the order of $\mathscr{L}$ at $(\alpha(p), f)$ is 1 for any $f$. We denote by $\Gamma$ the pseudo-group on $\mathbb{R}_{*}^{2}$ determined by the system $\left\{u, v, y \cdot \frac{\partial x}{\partial u}, y\right.$. $\left.\frac{\partial x}{\partial v}, \frac{D(x, y)}{D(u, v)}\right\}$. Then $\Gamma$ is complete at any $(z, 1), z \in \boldsymbol{R}_{*}^{2}$. By Theorem 6.1 , the system of differential equations

$$
\left\{\begin{array}{l}
y \cdot \frac{\partial x}{\partial u}=\alpha(u, v) \\
y \cdot \frac{\partial x}{\partial v}=\beta(u, v) \\
\frac{D(x, y)}{D(u, v)}=\gamma(u, v)
\end{array}\right.
$$

is $\Gamma$-automorphic if it possesses a solution. The integrability condition of $(*)^{\prime}$ is $\frac{\partial \alpha}{\partial v}-\frac{\partial \beta}{\partial u}=\gamma$. Since the 1-st and the 2-nd equalities induce the 3-rd equality by differentiation, we can see that the solution space of (*) is equal to that of $(*)^{\prime}$. Therefore $(*)$ is $\Gamma$-automorphic.

For two systems of differential equations

$(*)_{i}$

$$
\left\{\begin{array}{l}
y \cdot \frac{\partial x}{\partial u}=\alpha_{i}(u, v), \\
y \cdot \frac{\partial x}{\partial v}=\beta_{i}(u, v) \quad(i=1,2),
\end{array}\right.
$$

we assume that there is a number $c$ such that $\alpha_{2}=c \cdot \alpha_{1}$ and $\beta_{2}=c \cdot \beta_{1}$ and such that $|c-1|$ is sufficiently small. Then we shall prove that 
$(*)_{1}$ and $(*)_{2}$ are isomorphic.

We shall calculate the normalizer $\mathcal{N}(\mathscr{L})$ of $\mathscr{L}$. From the equality $\left[A(x, y) \cdot \frac{\partial}{\partial x}+B(x, y) \cdot \frac{\partial}{\partial y}, \xi(x) \cdot \frac{\partial}{\partial x}-\xi^{\prime}(x) \cdot y \cdot \frac{\partial}{\partial y}\right]=\eta(x) \cdot \frac{\partial}{\partial x}-\eta^{\prime}(x) \cdot y \cdot$ $\frac{\partial}{\partial y}$, we get the equalities

(1) $\xi^{\prime} \cdot A-\xi \cdot \frac{\partial A}{\partial x}+\xi^{\prime} \cdot y \cdot \frac{\partial A}{\partial y}=\eta$,

(2) $\xi^{\prime} \cdot y \cdot \frac{\partial B}{\partial y}-\xi^{\prime \prime} \cdot y \cdot A-\xi^{\prime} \cdot B-\xi \cdot \frac{\partial B}{\partial x}=-\eta^{\prime} \cdot y$.

From (1), we get

(3) $\eta^{\prime}=\xi^{\prime \prime} \cdot A-\xi \cdot \frac{\partial^{2} A}{\partial x^{2}}+\xi^{\prime \prime} \cdot y \cdot \frac{\partial A}{\partial y}+\xi^{\prime} \cdot y \cdot \frac{\partial^{2} A}{\partial x \partial y}$.

Therefore (2) and (3) induce the equality

$$
\xi\left(\frac{\partial B}{\partial x}+y \cdot \frac{\partial^{2} A}{\partial x^{2}}\right)+\xi^{\prime}\left(B-y \cdot \frac{\partial B}{\partial y}-y^{2} \cdot \frac{\partial^{2} A}{\partial x \partial y}\right)-\xi^{\prime \prime} \cdot y^{2} \cdot \frac{\partial A}{\partial y}=0 .
$$

Since $\xi$ is arbitrary, we get a system of differential equations

$$
\left\{\begin{array}{l}
\frac{\partial B}{\partial x}+y \cdot \frac{\partial^{2} A}{\partial x^{2}}=0, \\
B-y \cdot \frac{\partial B}{\partial y}-y^{2} \cdot \frac{\partial^{2} A}{\partial x \partial y}=0 \\
\frac{\partial A}{\partial y}=0 .
\end{array}\right.
$$

The 3-rd equation of $(* *)$ implies that $A(x, y)=\theta(x)$. Then $(* *)$ is replaced by the system of differential equations

$(* *)^{\prime}$

$$
\left\{\begin{array}{l}
\frac{\partial B}{\partial x}=-y \cdot \theta^{\prime \prime} \\
\frac{\partial B}{\partial y}=\frac{1}{y} \cdot B
\end{array}\right.
$$

The system $(* *)^{\prime}$ satisfies the integrability condition and we can easily see that a general solution of $(* *)^{\prime}$ is given by $-\left(\theta^{\prime}+a\right) \cdot y$ where $a$ is any constant number. This implies that the normalizer $\mathscr{N}(\mathscr{L})$ of $\mathscr{L}$ 
is generated by all local vector fields of the form $\theta(x) \frac{\partial}{\partial x}-\left(\theta^{\prime}+a\right) \cdot y \cdot \frac{\partial}{\partial y}$ where $\theta(x)$ is any local analytic function and $a$ is any constant.

Let $\phi_{t}\left(\right.$ resp. $\left.\psi_{t}\right)$ be the local 1-parameter group of local transformations induced from $a \cdot y \cdot \frac{\partial}{\partial y}\left(\operatorname{resp} . \theta(x) \cdot \frac{\partial}{\partial x}-\left(\theta^{\prime}+a\right) \cdot y \cdot \frac{\partial}{\partial y}\right)$. Then we have

$$
D\left(\phi_{t}\right)=D\left(\psi_{t}\right)
$$

and

$$
\begin{aligned}
& \left(\phi_{t}^{(1)}\right) * y \cdot \frac{\partial x}{\partial u}=e^{a \cdot t} \cdot y \cdot \frac{\partial x}{\partial u} \\
& \left(\phi_{t}^{(1)}\right)^{*} y \cdot \frac{\partial x}{\partial v}=e^{a \cdot t} \cdot y \cdot \frac{\partial x}{\partial v} \\
& \left(\phi_{t}^{(1)}\right)^{*} \frac{D(x, y)}{D(u, v)}=e^{a \cdot t} \cdot \frac{D(x, y)}{D(u, v)} .
\end{aligned}
$$

This implies that there exists an element $D(\phi) \in D(\mathscr{N}(\Gamma))$ such that $\left(\alpha_{2}\right.$, $\left.\beta_{2}, \gamma_{2}\right)=D(\phi) \circ\left(\alpha_{1}, \beta_{1}, \gamma_{1}\right)$. Therefore, by Theorem 7.1, $(*)_{1}^{\prime}$ and $(*)_{2}^{\prime}$ are isomorphic, that is, $(*)_{1}$ and $(*)_{2}$ are isomorphic.

\section{References}

[1] Kuranishi, M., Lectures on exterior differential systems, Tata Inst. Fund. Res., Bombay, 1962.

[2] Lie, S., Verwertung des Gruppenbegriffes für Differentialgleichungen. I, Leipz. Ber., 1895.

[3] — Über Differential Invarianten, Math. Ann., 24 (1884), 537-578.

[4] Singer, I. M. and Sternberg, S., The infinite groups of Lie and Cartan, $J$. Analyse Math., 15 (1965), 1-114.

[5] Ueno, K., Automorphic Systems and Lie-Vessiot Systems, Publ. RIMS, Kyoto Univ. 8 (1972), 311-334.

[6] Vessiot, E., Sur l'intégration des systèmès différentiels qui admettent des groupes continus de transformations, Acta Math., 28 (1904), 307-350. 